\title{
Home or Residential Care? The Role of Behavioral and Psychosocial Factors in Determining Discharge Outcomes for Inpatients with Huntington's Disease
}

\author{
Fiona Fisher*, Sophie Andrews, Andrew Churchyard and Susan Mathers \\ Calvary Health Care Bethlehem, Caulfield, VIC, Australia
}

\begin{abstract}
.
Background: The progressive nature of Huntington's disease (HD) means that families often struggle to cope with increasing care needs of their affected family member. As a result, individuals with HD are likely to be at risk of hospitalization and subsequent early placement into residential care facilities.

Objective: To explore which factors were associated with early residential care placement in a group of hospitalized patients with HD.

Methods: A retrospective, systematic medical file audit of a neurological inpatient unit was conducted. Fifty-nine patients with HD were admitted from the community between January 2008 and December 2011; of these, 31 patients were discharged home while 28 patients required discharge to a residential care facility. These two groups were compared on a range of demographic, clinical and psychosocial variables identified as precipitating the hospital admission. Group comparisons were performed using $t$-tests and chi-square tests with Bonferroni correction for multiple comparisons.

Results: A higher proportion of men were placed in residential care than were able to return home $(p=0.045)$; and the group placed in residential care had longer inpatient hospital stays $(p<0.001)$. Groups did not differ in age, medications or disease duration. We found that psychosocial difficulties $(p<0.001)$ and behavioral problems $(p=0.001)$, but not physical, cognitive, or psychiatric factors, significantly differentiated the groups.

Conclusions: Patients with HD discharged to residential care were more likely to have psychosocial and behavioral problems, and lengthy hospital stays. These findings indicate the need for community-based psychosocial and behavior management interventions aimed at preventing residential care admissions for persons with HD.
\end{abstract}

Keywords: Huntington's disease, social support, behavior, residential facilities, institutionalization, hospitalization

HD is an uncommon hereditary autosomal dominant neurodegenerative disorder characterized by involuntary movements, psychiatric changes, and cognitive decline [1]. This disorder causes an array of physical, psychiatric, cognitive and behavioral symptoms that

${ }^{*}$ Correspondence to: Dr. Fiona Fisher, Calvary Health Care Bethlehem, 476 Kooyong Rd Caulfield, VIC 3162, Australia. Tel.: +61 409956586; Fax: +61 039595 3335; E-mail: fisher.fiona@ gmail.com. interact in a disabling manner. The progressive nature of the disease means that families often struggle to cope with increasing care needs of their affected family member. As a result, individuals with HD are likely to be at risk of hospitalization and subsequent early placement into residential care facilities.

An Australian study surveyed residential aged care facilities in the state of Victoria [2]. Six hundred and twenty-six of 800 facilities responded. Survey results 
showed that 330 residents were under the age of 60 with $29 \%$ of persons diagnosed with a progressive neurological disease. Of this group, HD was the second most prominent diagnostic group behind multiple sclerosis, consistent with the lower prevalence of HD. For younger persons with acquired and progressive conditions, hospitals are the most frequent source of admission to residential aged care settings, with patients often spending extended periods in hospital prior to placement in residential aged care [2-4].

To date, few studies have examined the types of factors that lead to accommodation breakdown and residential care placement for persons with HD. Nance and Sanders [5] conducted a retrospective review of demographic and clinical characteristics of people with HD living in residential care facilities in the state of Minnesota, U.S.A. Ninety-seven medical files were reviewed. This study characteristed the "average newly institutionalized" person with HD as approximately 45 years old, symptomatic for at least 10 years, not currently married, and a high school graduate. It was also reported that on average, males were younger than females when admitted to residential care, but both genders had a similar disease duration. An absence of caregivers, or inability of caregivers to continue providing supporting at home were identified as common reasons for admission to residential care. An "inability to manage behavior" was identified as the most common reason for residents with HD to be transferred between residential care facilities. While the authors were able to identify a range of factors that were present following admission to residential care facilities, file audit data was not specific to the time of admission (i.e. behavioral difficulties were deemed present if noted "anytime" within chart notes). Furthermore, motor, cognitive, psychiatric and psychosocial factors were not directly explored.

Wheelock et al. [6] conducted a large international cross-sectional database study using the Huntington's Study Group Unified Huntington Disease Rating Scale (UHDRS) Database. The sample comprised a large cohort of persons with HD (2842 residing at home; 228 residing in residential care facilities) collected over a five-year period (1994-1999) to identify factors that predicted residential care placement. Of these, a subset of 87 participants who were followed longitudinally transitioned from living at home to living in institutionalized care during initial and follow-up time points. The average length of follow up was 1.89 years. Selected UHDRS items were used to examine motor function, psychiatric and behavioral symptoms. Motor dysfunction, psychiatric symptoms, and the presence of aggression and irritability were included as possible predictor variables. Results revealed that motor dysfunction alone, not psychiatric or behavioral symptoms, predicted residential care placement. Authors noted this finding as surprising given the significantly higher prevalence of both psychiatric symptoms and behavior disturbance in those placed in residential care facilities compared to those living at home. The authors also suggested that those with severe psychiatric and behavior disturbance may have been admitted to psychiatric facilities rather than mainstream residential care facilities, and so may not have been appropriately captured in the study.

In another large retrospective database audit, Rosenblatt et al. [7] examined a subset of patient data from the Baltimore Huntington's Disease Center database. During longitudinal follow-up at the center, 88 patients with HD were found to have transitioned from the community to long term residential care. This group was compared to 712 patients with HD who had no record of institutionalization. In this instance, the Quantified Neurological Examination was used to measure motor function. Behavior was examined by unpublished irritability and apathy scales used within the clinic while the Hamilton Depression Inventory was used as a measure of psychiatric symptomology. Consistent with Wheelock et al. [6], motor symptoms predicted placement into long term residential care. In addition, functional ability, and to a lesser extent, cognitive impairment (as measured by the MMSE) were also significant predictors. Again, psychiatric symptoms were not found to predict placement.

While the above database studies comprised large samples, the factors available for examination were restricted. For example, only limited psychosocial variables were available for analysis. Caregiver variables were also not included, despite caregiver stress being identified as key factor in the need for residential care placement in other neurodegenerative conditions such as Alzheimer's disease $[8,9]$. In addition, the trigger for placement in residential care did not necessarily coincide chronologically with the database time points.

The current project comprised a retrospective, systematic medical file audit. The aim was to identify factors leading to hospital admission that distinguished between those patients with HD who were able to be discharged home, and those who required discharge to residential care. Given that previous research has consistently identified motor dysfunction as predictive of residential care placement, it was anticipated that motor dysfunction (i.e. decreased mobility and increased falls) would be more prevalent in HD 
patients requiring discharge to a residential facility than patients discharged home. It was also anticipated that both caregiver- and patient-related psychosocial factors would differentiate between our two patient groups.

\section{METHOD}

\section{Sample characteristics}

This cohort study comprised data collected from a retrospective medical file audit of patient files from Calvary Health Care Bethlehem (CHCB). CHCB is a specialist Australian, statewide provider of multidisciplinary health services to patients with progressive neurological conditions. As well as providing specialist multidisciplinary outpatient HD clinics, CHCB has a 30-bed progressive neurology ward. HD patients occupy up to one third of inpatient beds and are admitted to the inpatient ward for both crisis and planned admissions. Patients are referred for medication review, symptom management and terminal care. As well as providing medical and allied health interventions for physical HD symptoms, patients are admitted for management of cognitive, psychiatric and behavioural sequelae. Inpatients are only transferred to acute psychiatric facilities in rare cases where individuals display signs of acute psychosis and cannot be managed within a sub-acute setting.

Medical files of all patients with a diagnosis of HD admitted to the CHCB inpatient progressive neurological ward between January 2008 and December 2011 were included in the audit. Ninety-seven patients with HD were admitted during this time. Of those, 32 patients were admitted to the inpatient unit from a residential facility and were discharged back to that residential facility. These medical files were excluded from further analysis. One patient was admitted for terminal care and died whilst an inpatient. Four patients became acutely unwell and required transfer to an acute hospital facility, and one patient was transferred to a rural hospital for subsequent placement. These patients were also excluded from the analysis. Fifty-nine HD patients were admitted from the community (i.e. from their home). Of these, 31 patients were discharged home while 28 patients were unable to return home and were discharged to a residential care facility. These two groups were compared in the current study. Ethical approval was obtained from the Calvary Health Care Bethlehem Human Research Ethics and Ethics Committee.

\section{Materials and procedure}

A structured file audit tool was developed to aid in the extraction of demographic and psychosocial information, and clinical variables identified as precipitating the admission to the neurological inpatient unit. Audit variables were guided by previous literature and expert clinical opinion. The CHCB specialist multidisciplinary clinical team and staff from the peak body Huntington's Victoria were interviewed, and a consensus of factors most commonly precipitating hospital admissions for HD patients were generated.

If a patient had multiple admissions during the audit timeframe, information pertaining to the latest admission was extracted from the medical file. Audit variables included a range of demographic variables. These included length of stay, age, gender, employment status, marital status, disease duration and admission Barthel Index. The Barthel Index is a well-validated measure of functional independence that measures activities of daily living and mobility across ten areas. Index scores range from 0-100 with higher scores associated with greater functional independence [10]. Demographic audit variables were a mix of continuous and categorical variables, while clinical and psychosocial variables were dichotomous categorical variables rated as present or absent. Clinical and psychosocial variables were defined as present if documented as a precipitating factor to the inpatient admission. These comprised physical factors (i.e., changes in mobility, swallowing, continence, communication, as well as weight loss, malnutrition and increased chorea, falls or sleep disturbance), functional factors (i.e., difficulties with showering, toileting, dressing, cooking, cleaning and accessing the community), cognitive factors and dementia, behavioral disturbance and psychiatric symptoms. Psychosocial factors were defined as patient-related (presence of either financial issues, breakdown in formal or informal supports and/or unstable accommodation) or caregiver-related (caregiver stress and/or caregiver health issues).

All medical files were rated by one of two clinicians. To evaluate inter-rater reliability of the file audit tool, five patient medical files were randomly selected and rated by both clinicians. Inter-rater agreement was 0.96 .

\section{Statistical analysis}

Two-group comparisons for categorical data were performed using the $\chi^{2}$ test with likelihood ratio test 
Table 1

Sample characteristics

\begin{tabular}{|c|c|c|c|}
\hline \multirow[t]{2}{*}{ Characteristics } & Home & Residential & \multirow[t]{2}{*}{$t$-test or $\chi^{2}$} \\
\hline & \multicolumn{2}{|c|}{$n(\%)$ or mean $(\mathrm{SD})$} & \\
\hline Length of admission & $26(51.54)$ & $144(92.57)$ & $t(41)=-5.96^{* *}$ \\
\hline Age at admission & $58.55(13.10)$ & $52.82(11.31)$ & N.S. \\
\hline Gender (males) & $13(41.9 \%)$ & $19(67.9 \%)$ & $\chi^{2}(1)=4.04^{*}$ \\
\hline Disease duration (years) & $7.2(4.46)$ & $5.20(4.86)$ & N.S. \\
\hline Barthel Index & $48.03(5.98)$ & $59.15(4.65)$ & N.S. \\
\hline \multicolumn{4}{|l|}{ Medications on admission } \\
\hline Antispychotic medication & $23(74.2 \%)$ & $22(78.6 \%)$ & N.S. \\
\hline Antidepressant medication & $17(54.8 \%)$ & $18(64.3 \%)$ & N.S. \\
\hline Mood Stabiliser & $12(38.7 \%)$ & $13(46.4 \%)$ & N.S. \\
\hline Benzodiazepine & $6(19.4 \%)$ & $7(25.0 \%)$ & N.S. \\
\hline Tetrabenazine & $10(32.3 \%)$ & $8(28.6 \%)$ & N.S. \\
\hline
\end{tabular}

${ }^{*} p<0.05 ;{ }^{* *} p<0.001$.

statistic, and independent sample $t$-tests were used for continuous data. All tests were two-tailed, with $\alpha<0.05$. A Bonferroni correction was applied to alpha levels to control for multiple comparisons. Bonferroni correction resulted in an adjusted alpha of 0.007 $(0.05 / 7)$ being applied to group comparisons between home and residential groups.

\section{RESULTS}

\section{Sample characteristics}

Sample characteristics for the two groups are included in Table 1. Inpatients discharged to a residential care facility had significantly longer hospital admissions than those discharged home $(p<0.001)$.

There was no difference between the groups on age at admission. The group discharged home ranged in age from 34 to 77 years, while the group discharged to residential care ranged in age from 30 to 74 years. There were a significantly higher proportion of males in the group discharged to residential care compared to the group discharged home. There was no difference between the groups with regard to disease duration in years, or medications on admission.

Barthel Index scores on admission revealed no significant group differences in functional independence (that is ability to perform activities of daily living), $t(52)=-1.47, p>0.05$. There were also no significant differences between groups in the number of hospital admissions in the 12 months prior to admission, $t(55)=-0.45, p>0.05$.

\section{Factors precipitating hospital admission}

As can be seen in Table 2, a number of admission factors were identified in both the home and residential groups. Chi square analyses were used to examine the associations between the category of difficulties patients experienced on admission, and subsequent discharge destination. Behavior difficulties significantly differentiated between HD patients placed in residential care and those able to return home, $\chi^{2}(1)=10.80, p=0.001$, with an odds ratio suggesting that patients with behavioral difficulties were 6.17 times more likely to be discharged to a residential care facility than discharged home. Within the behavioral variables examined, aggressive behavior occurred most frequently in the residential care group $(35.7 \%)$ in comparison to the home group (3.2\%).

There was also a significant difference between groups with regard to the presence of psychosocial issues, $\chi^{2}(1)=19.51, p<0.001$, with an odds ratio suggesting that patients experiencing financial difficulties, and breakdown in accommodation and social support were 19 times more likely to be discharged to residential care facility than discharged home. While we note that a higher proportion of caregiver stress and health issues in the group discharged to residential care, this difference was not significant once the Bonferroni correction was applied, $\chi^{2}(1)=4.95, p=0.026$. There was no difference between groups with regard to the frequency of physical difficulties, $\chi^{2}(1)=0.28, p=0.60$, or functional difficulties, $\chi^{2}(1)=1.33, p=0.25$. Further, no differences in cognitive function $\chi^{2}(1)=1.91$, $p=0.167$, or psychiatric factors $\chi^{2}(1)=2.75, p=0.10$, were detected between the groups.

\section{DISCUSSION}

This study explored the impact of precipitating hospital admission factors on discharge outcome in a group of HD patients. We found that HD patients who were admitted to hospital with psychosocial and behavioral 
Table 2

Frequency of identified factors precipitating hospital admission

\begin{tabular}{|c|c|c|}
\hline Factors & $\begin{array}{l}\text { Home } \\
n=31\end{array}$ & $\begin{array}{c}\text { Residential } \\
n=28\end{array}$ \\
\hline Physical difficulties & $27(87.1 \%)$ & $23(82.1 \%)$ \\
\hline Chorea & $14(45.2 \%)$ & $9(32.1 \%)$ \\
\hline Mobility changes & $5(16.1 \%)$ & $7(25.0 \%)$ \\
\hline Increased falls & $15(48.4 \%)$ & $12(42.9 \%)$ \\
\hline Swallowing difficulties & $10(32.3 \%)$ & $7(25.0 \%)$ \\
\hline Communication issues & $1(3.2 \%)$ & $0(0.0 \%)$ \\
\hline Continence issues & $0(0.0 \%)$ & $1(3.6 \%)$ \\
\hline Weight loss & $3(9.7 \%)$ & $4(14.3 \%)$ \\
\hline Malnutrition & $0(0.0 \%)$ & $1(3.6 \%)$ \\
\hline Sleep disturbance & $1(3.2 \%)$ & $4(14.3 \%)$ \\
\hline Functional difficulties & $5(16.1 \%)$ & $8(28.6 \%)$ \\
\hline Problems toileting & $4(12.9 \%)$ & $5(17.9)$ \\
\hline Problems showering & $4(12.9 \%)$ & $7(25 \%)$ \\
\hline Problems dressing & $3(9.7 \%)$ & $5(17.9 \%)$ \\
\hline Problems cooking & $3(9.7 \%)$ & $8(28.6 \%)$ \\
\hline Problems cleaning & $3(9.7 \%)$ & $6(21.4 \%)$ \\
\hline Problems accessing community & $3(9.7 \%)$ & $8(28.6 \%)$ \\
\hline Cognitive dysfunction & $9(29 \%)$ & $13(46.4 \%)$ \\
\hline Behavioral disturbance & $7(22.6 \%)$ & $18(64.3)^{*}$ \\
\hline Apathy/adynamia & $4(12.9 \%)$ & $5(17.9 \%)$ \\
\hline Rigid and fixed behaviors & $3(9.7 \%)$ & $5(17.9 \%)$ \\
\hline Impulsiveness & $0(0.0 \%)$ & $4(14.3 \%)$ \\
\hline Irritable/agitated behavior & $5(16.1 \%)$ & $10(35.7 \%)$ \\
\hline Aggression & $1(3.2 \%)$ & $10(35.7 \%)$ \\
\hline Disinhibited behavior & $1(3.2 \%)$ & $3(10.7 \%)$ \\
\hline Psychiatric disturbance & $8(25.8 \%)$ & $13(46.4 \%)$ \\
\hline Issues with depressed mood & $5(16.1 \%)$ & $8(28.6 \%)$ \\
\hline Anxiety issues & $3(9.7 \%)$ & $6(21.4 \%)$ \\
\hline Obsessive compulsive disorder & $2(6.5 \%)$ & $2(7.1 \%)$ \\
\hline Psychosis present & $0(0.0 \%)$ & $3(10.7 \%)$ \\
\hline Psychosocial patient related factors & $2(6.5 \%)$ & $16(57.1 \%)^{*}$ \\
\hline Financial issues & $2(6.5 \%)$ & $8(28.6 \%)$ \\
\hline Breakdown in formal supports & $0(0.0 \%)$ & $5(17.9 \%)$ \\
\hline Breakdown in informal supports & $0(0.0 \%)$ & $9(32.1 \%)$ \\
\hline Issues with accommodation & $0(0.0 \%)$ & $20(71.4 \%)$ \\
\hline Psychosocial caregiver related factors & $11(35.5 \%)$ & $18(64.3 \%)$ \\
\hline Caregiver stress & $11(35.5 \%)$ & $17(60.7 \%)$ \\
\hline Caregiver health issues & $1(3.2 \%)$ & $5(17.9 \%)$ \\
\hline
\end{tabular}

${ }^{*} p<0.007$ Bonferroni adjusted.

problems were more likely to require placement in residential care. In contrast, there was no difference between groups with regard to physical symptoms, functional difficulties, cognitive dysfunction or psychiatric symptoms.

Our study has highlighted the importance of psychosocial and behavioral factors in differentiating between HD patients requiring placement in residential care with those able to return home following an inpatient hospital stay. In contrast to the previous research of Rosenblatt et al. [7] and Wheelock et al. [6], the current study found that the presence of motor symptoms did not differentiate groups. That is, motor symptoms (increased falls, reduced mobility and increased chorea) were documented as factors precipitating admission for a similar number of patients in each group and did not appear disproportionally in the group of patients requiring residential care placement. This is consistent with the lack of group differences evident on our measure of overall functional disability. Unfortunately, UHDRS motor scores were not completed on admission to $\mathrm{CHCB}$, meaning this gold standard measurement for rating physical disability was not available for analysis.

Our finding that behavioral disturbance significantly differentiated the home and residential groups is consistent with the findings of Nance and Sanders [5] but only partially supports the more recent findings of Wheelock et al. [6] who found that despite a higher prevalence of aggressive and irritable behaviors in their nursing home sample, these behaviors did not predict placement. Our findings are in contrast to those of Rosenblatt et al. [7] who found no association between levels of apathy and irritability, and residential care placement. These contrasting findings may be explained by the restricted range of behavioral variables available for analysis in these studies. For example, Wheelock and colleagues' use of the UHDRS behavior scale may have limited the detection of the full range of behavior symptoms common in HD. Behavioral symptoms were detected in $64.3 \%$ of our residential care sample, compared to approximately $26 \%$ of that of Wheelock et al. It is recognized that a limitation of the UHDRS is its reduced sensitivity in detecting behavioral symptoms common in HD [11]. Similarly, Rosenblatt et al. measured behaviors related to apathy and irritability, but did not sample other key behavioral domains, such as aggression and impulsive behavior.

Another possible explanation for the discrepancies in behavioral findings may be related to methodological differences. Biases inherent in the sampling of HD patients were raised as a potential limitation by both Wheelock et al. and Rosenblatt et al. It was suggested that HD patients with more severe psychiatric and behavioral disturbances might have been disproportionally represented in the group of participants who were lost to follow up in their studies.

Psychosocial factors (i.e., financial difficulties, breakdowns in both informal and formal supports, and issues with current accommodation) were found to be significantly more prevalent in the group requiring residential care placement. While this finding is consistent with previous research in general dementia populations 
$[8,12,13]$, this is the first in the HD literature to identify psychosocial difficulties as precipitating factors to residential care placement. This is an important finding given that psychosocial problems occur frequently in HD [14].

With regard to caregiver factors, despite higher frequencies of caregiver stress and health issues in the group requiring residential placement (i.e. $64.3 \%$ compared to $35.5 \%$ in the home group), caregiver factors were not found to differentiate groups. However, the small sample size and conservative approach to statistical analysis (i.e. use of Bonferroni adjustment for multiple comparisons) may account for this nonsignificant finding. Given that published research in dementia care has identified caregiver stress as a significant predictive factor of residential care placement $[8$, 9], further examination of the impact on caregiver factors in residential care placement in HD is warranted.

The main limitation of our study is that data were extracted retrospectively from medical records. Some information was not consistently available (e.g., CAG repeat length), limiting the variables that could be explored. Future research could address this issue by conducting large prospective studies. This would also permit more in-depth exploration of psychosocial, caregiver and behavioral precipitants of residential care placement. In addition, data for this retrospective medical file audit was obtained from a specialist progressive neurological unit and thus findings may not generalize to the wider HD population. Although the sample size was relatively small, given the rare nature of HD it is unlikely that large enough datasets could be obtained from mainstream hospital settings.

In conclusion, HD patients discharged to residential care were more likely to have psychosocial and behavioral problems, compared to those able to be supported in the community. In Australia, local support services to assist people with disabilities to complete personal, domestic and community activities of daily living are available including home help, shopping assistance and meal delivery. Without a live-in carer or family member, these services alone are often inadequate to sustain a person with more advanced HD in the community. The progressive nature of HD does not fit neatly within the disability service framework, which often fails to track the changing needs of a person with a neurodegenerative disease. The young age of disease onset prevents access to dementia support services that cater to those over the age of 65 years. At present, specialist services for people with HD are limited. Most people with HD who are admitted to hospital are managed on general medical or neurological wards, even when psychiatric symptoms or behavior disturbance are prominent. With this in mind, the utility of community-based interventions for psychosocial issues and behavioral difficulties (e.g. case management, counseling support for caregivers, behavior management interventions for behaviors of concern) warrants further exploration in HD. Research has successfully implemented interventions to reduce breakdowns of support networks, better manage behavioral difficulties and delay admission to residential care in other neurological disease groups [15-17]. By trialing community-based interventions targeting psychosocial functioning and management of behavior difficulties in the HD population, similar positive findings may be expected. Given HD patients discharged to residential care had lengthy (and therefore costly) hospital stays, interventions aimed at prolonging community living would not only promote improved quality of life, but also have the potential to significantly reduce health care expenditure.

\section{ACKNOWLEDGMENTS}

The authors would like to thank the multidisciplinary team at $\mathrm{CHCB}$ and Huntington's Victoria for supporting this research. We would also like to acknowledge the Collier Charitable Fund for providing the funding support for this research. Finally, we would like to thank Professor Julie Stout for her guidance and Associate Professor Jacinta Douglas for her statistical advice and input during review.

\section{DISCLOSURE OF INTERESTS}

The authors report no conflicts of interest.

\section{REFERENCES}

[1] Roos RAC. Huntington's disease: A clinical review. Orphanet Journal of Rare Diseases. 2010;5(1):40.

[2] Winkler D, Farnworth L, Sloan S. People under 60 living in aged care facilities in Victoria. Aust Health Rev. 2006;30(1):100-8.

[3] Winkler D, Farnworth L, Sloan S, Brown T. Young people in aged care: Progress of the current national program. Aust Health Rev. 2011;35(3):320-6.

[4] Winkler D, Sloan S, Callaway L. People under 50 with acquired brain injury living in residential aged care. Brain Impairment. 2010;11(3):299.

[5] Nance MA, Sanders G. Characteristics of individuals with Huntington disease in long-term care. Mov Disord. 1996;11(5):542-8.

[6] Wheelock VL, Tempkin T, Marder K, Nance M, Myers RH, Zhao H, et al. Predictors of nursing home placement in Huntington disease. Neurology. 2003;60(6):998-1001. 
[7] Rosenblatt A, Kumar BV, Margolis RL, Welsh CS, Ross CA. Factors contributing to institutionalization in patients with Huntington's disease. Mov Disord. 2011;26(9):1711-6.

[8] Yaffe K, Fox P, Newcomer R, Sands L, Lindquist K, Dane $\mathrm{K}$, et al. Patient and caregiver characteristics and nursing home placement in patients with dementia. JAMA. 2002;287(16):2090-7.

[9] Banerjee S, Murray J, Foley B, Atkins L, Schneider J, Mann A. Predictors of institutionalisation in people with dementia. J Neurol Neurosurg Psychiatry. 2003;74(9):1315-6.

[10] Mahoney FI. The Barthel Index. Maryland State Med J. 1965;14:61-5.

[11] Craufurd D, Thompson JC, Snowden JS. Behavioral changes in Huntington disease. Neuropsychiatry Neuropsychol Behav Neurol. 2001;14(4):219-26

[12] Luppa M, Riedel-Heller SG, Luck T, Wiese B, van den Bussche H, Haller F, et al. Age-related predictors of institutionalization: Results of the German study on ageing, cognition and dementia in primary care patients (AgeCoDe). Soc Psychiatry Psychiatr Epidemiol. 2012:1-8.
[13] Haupt M, Kurz A. Predictors of nursing home placement in patients with Alzheimer's disease. Int J Geriatr Psychiatry. 1993;8(9):741-6.

[14] Pender NP, Koroshetz WJ. Huntington's disease. In: Hardiman O, Doherty CP, editors. Neurodegenerative Disorders. London: Springer; 2011. p. 167-79.

[15] Brodaty H, Green A, Koschera A. Meta-analysis of psychosocial interventions for caregivers of people with dementia. Am J Geriatr Psychiatry. 2003;51(5):657-64.

[16] Marriott A, Donaldson C, Tarrier N, Burns A. Effectiveness of cognitive-behavioural family intervention in reducing the burden of care in carers of patients with Alzheimer's disease. Br J Psychiatry. 2000;176(6):557-62

[17] Mittleman MS, Haley W, Clay O, Roth D. Improving caregiver well-being delays nursing home placement of patients with Alzheimer's disease. Neurology. 2006;67(9):1592-9. 